\title{
Optimum aerofoil parameterization for aerodynamic design
}

\author{
R. W. Derksen ${ }^{1} \&$ T. Rogalsky ${ }^{2}$ \\ ${ }^{I}$ Department of Mechanical and Manufacturing Engineering, \\ University of Manitoba, Winnipeg, Canada \\ ${ }^{2}$ Mathematics Department, Canadian Mennonite University, Winnipeg, \\ Canada
}

\begin{abstract}
The numerical search for the optimum shape of an aerofoil is of great interest for aircraft and turbomachine designers. Unfortunately, this process is very computationally intense and can require a large number of individual flow field simulations resulting in very long CPU run times. One of the core issues that the designer must deal with is how to describe the shape of the airfoil. Clearly, we can not treat the profile on a point by point basis as the problem would have an infinite number of degrees of freedom. Hence the typical practice is to resort to using a series of curves, such as polynomials and Bezier curves, to describe the profile. This typically reduces the number of degrees of freedom to a much smaller, manageable number. The influence of the selection of the parameterization on the optimization has received relatively little consideration to date. We can anticipate that some parameterizations will be less suitable for describing the profile shape and may result in slower convergence times.

Our paper will discuss a new airfoil parameterization, Bezier-PARSEC, that was developed to extend and improve the typical Bezier parameterization found in use. This parameterization was found to fit the known shape of a wide range of existing airfoil profiles as well as resulting in accelerated convergence for aerodynamic optimization using Differential Evolution. Our presentation will present the development and details of the Bezier-PARSEC parameterization and provide evidence that the parameterization is suitable and accelerates convergence.
\end{abstract}

Keywords: aerodynamic optimization, airfoil parameterization, Bezier curves. 


\section{Introduction}

The profiles of most airfoils given in the literature are described by a tabulated vector of 50 to 80 coordinate pairs located on the profile surface as demonstrated by NACA compilation given by Abbot and von Doenhoff [1]. The practical demands of aerodynamic optimization on CPU time require us to search for an effective parameterization that contains fewer degrees of freedom. Four main objectives must be considered in developing an airfoil parameterization: minimizing the number of degrees of freedom as possible, the parameterization should be able to represent a wide range of existing airfoils, the parameters should be simple to formulate and impose, and finally, the parameterization should result in effective and efficient optimization.

The following work describes a new method of representing an airfoil that is an extension of a Bezier parameterization previously used by the authors [2] but uses aerodynamic parameters. These are similar to the PARSEC parameters developed by Sobieczky [3].

\section{Bezier-PARSEC parameterization}

A Bezier parameterization is determined by its control points which are physical points in the plane. The first and last control points are the initial and terminal point on the curve itself. However the other control points need not be on the curve even though they determine the shape of the curve. As such a Bezier parameterization of an airfoil has control points that are only indirectly determined by the underlying aerodynamics. It is desirable to have the parameters such as leading edge radius, trailing wedge angle, and so on that do have physical relevance to the flow, such as that demonstrated by Sobieczky [3, 4]. Oyama et al. [5] showed that this type of parameterization improved the robustness and convergence speed for aerodynamic optimization, which makes it more suitable for optimization using genetic algorithms. The superior performance of PARSEC parameterization is likely due to its ability to minimize epistasis - the nonlinear manner in which the objective function is dependent on the design parameters. Typically a reduction in nonlinear interaction of the parameters will enable the optimizer to converge more quickly.

This motivated the development of a combined Bezier-PARSEC (BP) parameterization to utilize the advantages of both the Bezier and PARSEC parameterizations. The BP parameters are aerodynamically oriented, and allow us to obtain the Bezier control points of four separate curves. These curves describe the leading and trailing portions of the camber line, and the leading and trailing portion of the thickness distributions. Each curve is ultimately a Bezier curve and will be denoted by BP ijkl, where $i$ and $j$ are the order of the leading and trailing thickness curve, and $\mathrm{k}$ and $\mathrm{l}$ are the order of the leading and trailing camber curves.

The parameters are: leading edge radius $-r_{l e}$, trailing camber line angle $-\alpha_{t e}$, trailing wedge angle $-\beta_{t e}$, trailing edge vertical displacement $-z_{t e}$, leading edge 
direction $\gamma_{l e}$, location of the camber crest - $\left(x_{c}, y_{c}\right)$, curvature of the camber crest $-\kappa_{c}$, position of the thickness crest $-\left(x_{t}, y_{t}\right)$, curvature of the thickness crest $-\kappa_{t}$, the half thickness of the trailing edge - $d z_{t e}$, and several Bezier variable, $b_{0}, b_{2}, b_{8}, b_{15}$ and $b_{17}$. The detailed development of the BezierPARSEC curves is given by Rogalsky [6] and only the results will be given here.

\subsection{BP 3333 parameterization}

A BP 3333 parameterization uses third degree Bezier curves for all four curves used to define the airfoil. A third degree Bezier curve is given parametrically by

$$
x(u)=x_{0}(1-u)^{3}+3 x_{1} u(1-u)^{2}+3 x_{2} u^{2}(1-u)+x_{3} u^{3},
$$

and

$$
y(u)=y_{0}(1-u)^{3}+3 y_{1} u(1-u)^{2}+3 y_{2} u^{2}(1-u)+y_{3} u^{3},
$$

where $u$ is the parameter that runs from 0 at the beginning to 1 at its terminus. The BP 333 parameterization relies exclusively on the aerodynamic parameters there are no free Bezier points in BP 3333. The $x_{i}$ and $y_{i}$ are the Bezier control points, which are computed from the following:

\subsubsection{Leading edge thickness curve}

The control points are given by

$$
\begin{array}{ll}
x_{0}=0 & y_{o}=0 \\
x_{1}=0 & y_{1}=3 \kappa_{t}\left(x_{t}-b_{9}\right)^{2} / 2+y_{t} \\
x_{2}=b_{9} & y_{2}=y_{t} \\
x_{3}=x_{t} & y_{3}=y_{t}
\end{array}
$$

The parameter $b_{9}$ the root of

$$
\begin{aligned}
& 27 \kappa_{t}^{2} b_{9}^{4} / 4-27 \kappa_{t}^{2} x_{t} b_{9}^{3}+\left(9 \kappa_{t} y_{t}+81 \kappa_{t}^{2} x_{t}^{2} / 2\right) b_{9}^{2} \\
& \quad+\left(2 r_{l e}-18 \kappa_{t} x_{t} y_{t}-27 \kappa_{t}^{2} x_{t}^{3}\right) b_{9}+\left(3 y_{t}^{2}+9 \kappa_{t} x_{t}^{2} y_{t}+27 \kappa_{t}^{2} x_{t}^{4} / 4\right)=0
\end{aligned}
$$

within the bounds given by

$$
\max \left(0, x_{t}-\sqrt{-2 y_{t} / 3 \kappa_{t}}\right)<b_{9}<x_{t} .
$$

\subsubsection{Trailing edge thickness curve}

The control points are given by

$$
\begin{array}{ll}
x_{0}=x_{t} & y_{0}=y_{t} \\
x_{1}=2 x_{t}-b_{9} & y_{1}=y_{t} \\
x_{2}=1+\left[d z_{t e}-\left(3 \kappa_{t}\left(x_{t}-b_{9}\right)^{2} / 2+y_{t}\right)\right] \cot \left(\beta_{t e}\right) & y_{2}=3 \kappa_{t}\left(x_{t}-b_{9}\right)^{2} / 2+y_{t} \\
x_{3}=1 & y_{3}=d z_{t e}
\end{array}
$$




\subsubsection{Leading edge camber curve}

The control points are given by

$$
\begin{array}{ll}
x_{0}=0 & y_{0}=0 \\
x_{1}=b_{1} \cot \left(\gamma_{l e}\right) & y_{1}=b_{1} \\
x_{2}=x_{c}-\sqrt{2\left(b_{1}-y_{c}\right) / 3 \kappa_{c}} & y_{2}=y_{c} \\
x_{3}=x_{c} & y_{3}=y_{c}
\end{array}
$$

The parameter $b_{1}$ is computed from

$$
\begin{aligned}
b_{1} & =\left[16+3 \kappa_{c}\left(\cot \gamma_{l e}+\cot \alpha_{t e}\right)\left(1+z_{t e} \cot \alpha_{t e}\right)\right] /\left[3 \kappa_{c}\left(\cot \gamma_{l e}+\cot \alpha_{t e}\right)\right] \\
& \pm 4 \sqrt{16+6 \kappa_{c}\left(\cot \gamma_{l e}+\cot \alpha_{t e}\right)\left(1-y_{c}\left(\cot \gamma_{l e}+\cot \alpha_{t e}\right)+z_{t e} \cot \alpha_{t e}\right)}
\end{aligned}
$$

and must be within the following bounds

$$
0<b_{1}<y_{c} \text {. }
$$

\subsubsection{Trailing edge camber curve}

The control points are given by

$$
\begin{array}{ll}
x_{0}=x_{c} & y_{0}=y_{c} \\
x_{1}=x_{c}+\sqrt{2\left(b_{1}-y_{c}\right) / 3 \kappa_{c}} & y_{1}=y_{c} \\
x_{2}=1+\left(z_{t e}-b_{1}\right) \cot \left(\alpha_{t e}\right) & y_{2}=b_{1} \\
x_{3}=1 & y_{3}=z_{t e}
\end{array}
$$

\subsection{BP 3434 Parameterization}

A BP 3434 parameterization uses third degree Bezier curves for all of the leading edge curves and fourth degree Bezier curves for the both trailing curves used to define the airfoil. A fourth degree Bezier curve is given parametrically by

$$
x(u)=x_{0}(1-u)^{4}+4 x_{1} u(1-u)^{3}+6 x_{2} u^{2}(1-u)^{2}+4 x_{3} u^{3}(1-u)+x_{4} u^{4},
$$

and

$$
y(u)=y_{0}(1-u)^{4}+4 y_{1} u(1-u)^{3}+6 y_{2} u^{2}(1-u)^{2}+4 y_{3} u^{3}(1-u)+y_{4} u^{4} .
$$

\subsubsection{Leading edge thickness curve}

The control points are given by

$$
\begin{array}{ll}
x_{0}=0 & y_{o}=0 \\
x_{1}=0 & y_{1}=b_{8} \\
x_{2}=-3 b_{8}^{2} / 2 r_{l e} & y_{2}=y_{t} \\
x_{3}=x_{t} & y_{3}=y_{t}
\end{array}
$$

Here the parameter $b_{1}$ is subject to the following restriction

$$
0<b_{8}<\min \left(y_{t}, \sqrt{-2 r_{l e} x_{t} / 3}\right) .
$$




\subsubsection{Trailing edge thickness curve}

The control points are given by

$$
\begin{aligned}
& x_{0}=x_{t} \\
& x_{1}=\left(7 x_{t}+9 b_{8}^{2} / 2 r_{l e}\right) / 4 \\
& x_{2}=3 x_{t}+15 b_{8}^{2} / 4 r_{l e} \\
& x_{3}=b_{15} \\
& x_{4}=1
\end{aligned}
$$

$$
\begin{aligned}
& y_{0}=y_{t} \\
& y_{1}=y_{t} \\
& y_{2}=\left(y_{t}+b_{8}\right) / 2 \\
& y_{3}=d z_{t e}+\left(1-b_{15}\right) \tan \left(\beta_{t e}\right) \\
& y_{4}=d z_{t e}
\end{aligned}
$$

\subsubsection{Leading edge camber curve}

The control points are given by

$$
\begin{aligned}
& x_{0}=0 \\
& x_{1}=b_{0} \\
& x_{2}=b_{2} \\
& x_{3}=x_{c}
\end{aligned}
$$

$$
\begin{aligned}
& y_{0}=0 \\
& y_{1}=b_{0} \tan \left(\gamma_{l e}\right) \\
& y_{2}=y_{c} \\
& y_{3}=y_{c}
\end{aligned}
$$

\subsubsection{Trailing edge camber curve}

The control points are given by

$$
\begin{aligned}
& x_{0}=x_{c} \\
& x_{1}=\left(3 x_{c}-y_{c} \cot \left(\gamma_{l e}\right)\right) / 2 \\
& x_{2}=\left(-8 y_{c} \cot \left(\gamma_{l e}\right)+13 x_{c}\right) / 6 \\
& x_{3}=b_{17} \\
& x_{4}=1
\end{aligned}
$$

$$
\begin{aligned}
& y_{0}=y_{c} \\
& y_{1}=y_{c} \\
& y_{2}=5 y_{c} / 6 \\
& y_{3}=z_{t e}-\left(1-b_{17}\right) \tan \left(\alpha_{t e}\right) \\
& y_{4}=z_{t e}
\end{aligned}
$$

The parameterizations assume that the length of the airfoil has been normalized to unit length.

\subsection{Airfoil Representation}

The parameterization method must be able tow represent a wide range of airfoils if it is to contribute to a robust design algorithm. The authors compared the ability of Bezier and BP methods to reproduce 63 known airfoils: 40 NACA symmetric and asymmetric airfoils [7], 15 Eppler airfoils [8], and 8 low-speed airfoils [9]. Data for each of the selected airfoils consisted of an ordered array of coordinates for each profile.

The curves were fit to the coordinates using a modified airfoil design code that used Differential Evolution to find the parameters that minimized the $\ell_{2}$ - error norm between the representation and the given data points. The DE algorithm was rand-to-best/1/exp with $\mathrm{F}=0.85, \mathrm{CR}=1$, and a population of 150 members. The maximum number of generations permitted was 500 , however, in a few cases an additional run was used to obtain convergence. The convergence 
requirement was that the cost limit was less than 0.01 . For a unit chord length airfoil this corresponds to an average deviation of approximately $8 \times 10^{-4}$.

The following discussion will be limited to a few representative airfoils due to limited space.

\subsubsection{NACA Symmetric Airfoils}

The Bezier, BP 3333 and BP 3434 approximations converged for all of the symmetric airfoils. Typically, fewer that 3,000 approximations, or 20 generations were required to obtain convergence, with one exception, the BP 3333 parameterization of the NACA $67_{1}-015$. This indicates that matching the geometry of a known airfoil than for an inverse design using a Bezier parameterization [2].

The parameterization of the NACA 0008-34 airfoil will be presented in more detail. The curve fitting required the following; Bezier fit - 6,612 function evaluations with an average deviation of $1.06 \times 10^{-4}$, BP 3333 fit $-4,243$ function evaluations with an average deviation of $1.24 \times 10^{-4}$, and BP 3434 fit 6,180 function evaluations with an average deviation of $1.26 \times 10^{-4}$. Clearly, all of the parameterization methods could successfully approximate the shape of the symmetric profiles. The fit to the NACA 00008-34 is shown below, in Figure 1.
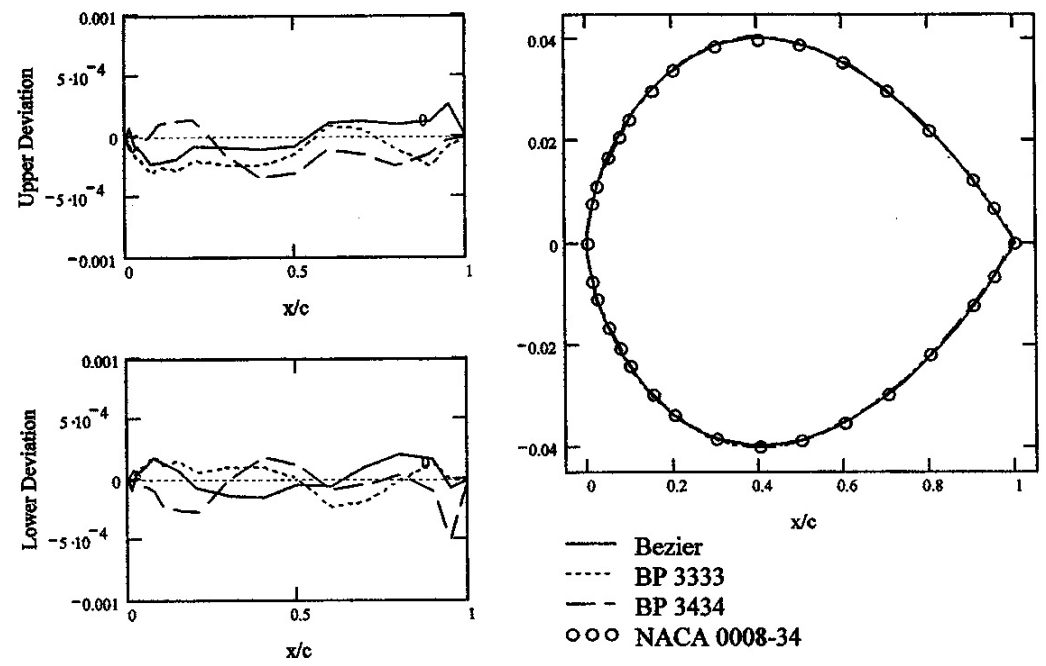

Figure 1: Representation of the NACA 0008-34 airfoil.

\subsubsection{NACA asymmetric airfoils}

The Bezier and BP 3434 were able to successfully reproduce each of the 20 asymmetric airfoils, with the BP 3333 failing to reproduce one, the NACA $744 \mathrm{~A} 315$ to the required tolerance. The average number of function evaluations for the successful representations are as follows: Bezier fit - 3,302, BP 3333 3,071 , and BP $3434-4,424$. A successful fit for each method is shown below, in Figure 2, for the NACA $63_{1}-212$ airfoil. 

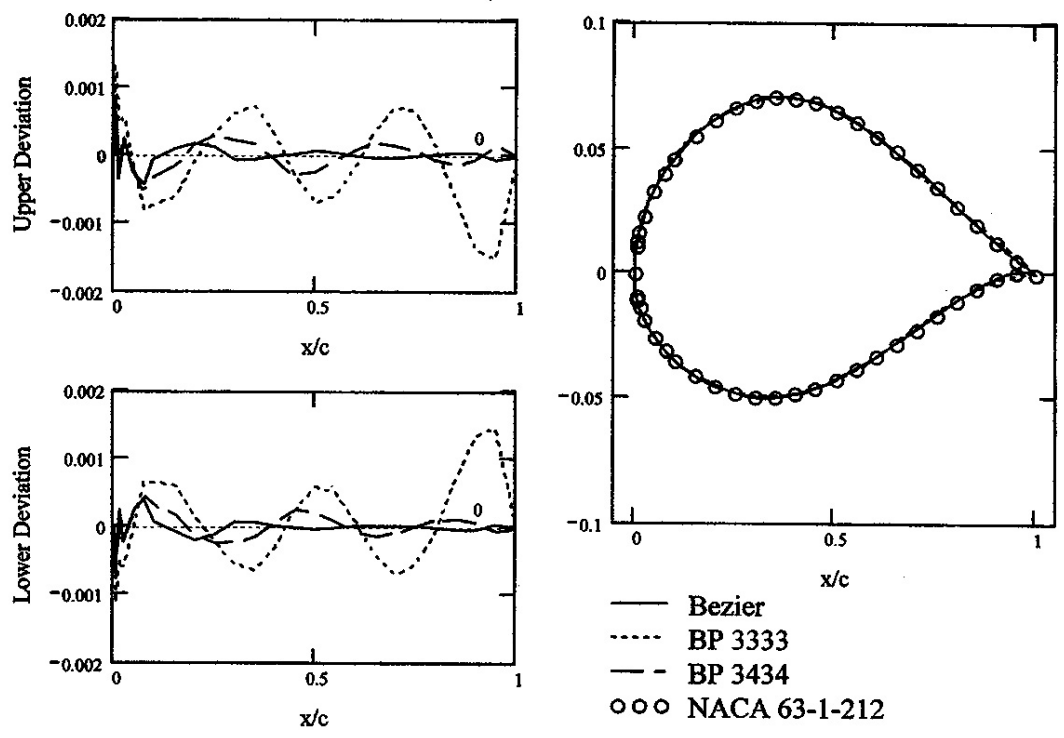

Figure 2: $\quad$ NACA 63-1-212 airfoil.

The failure of the BP 3333 parameterization for the NACA 747A315 was occurred near the trailing edge. This was primarily attributed to a lack of sufficient freedom on the trailing edge camber with minor issues with the trailing edge thickness profile. The NACA 747A315 has a sharp cusp at the trailing edge that is difficult to approximate. This was not an issue with the BP 3434 approximation as it has additional control points at the trailing edge.

\subsubsection{Eppler airfoils}

Eppler pursued the development of accurate theoretical methods to obtain inverse designs of airfoils with prescribed boundary layer characteristics. This has resulted in a catalogue of well established airfoils [8]. These airfoils represent a greater challenge due to their shape. The attempts to approximate these airfoils were less successful with the following success rates: Bezier $73 \%$, BP $3333-80 \%$, and BP $3434-90 \%$. The number of function evaluations for the successful approximations were: Bezier - 3,316, BP 3333 - 3,570, and BP $3434-4,870$. The resulting approximations for the E 266 airfoil are shown below in Figure 3.

The reasons for the failure to approximate the Eppler airfoils to the required accuracy are numerous, and include a lack of degrees of freedom, difficulty with airfoils that have a positive leading edge and negative trailing edge directions, and finite trailing edge thickness to name a few issues.

\subsubsection{Low-speed airfoils}

All of the methods were able to reproduce the selected set of low-speed airfoils to the desired tolerance. The average number of function evaluations required to 

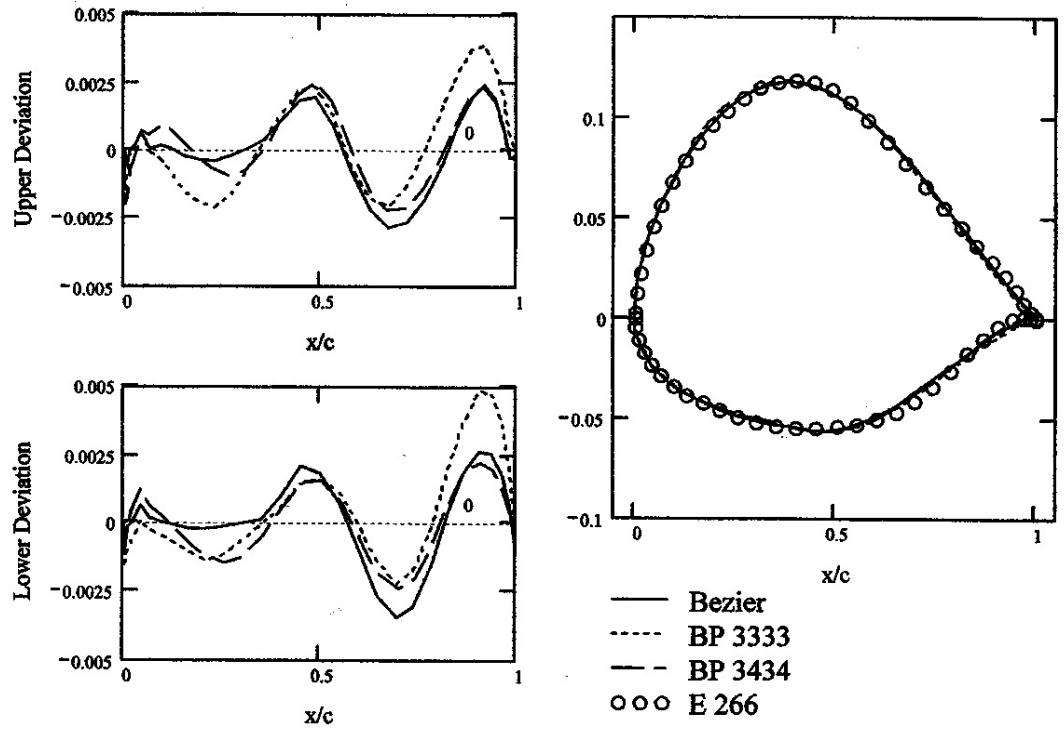

Figure 3: $\quad$ Eppler E 266 airfoil.

fit the airfoils were: Bezier - 5,483, BP 3333 - 8,834, and BP $3434-9,994$. A representative fit for the low-speed airfoils is given for the FX 74-CL5-140 MOD airfoil shown in Figure 4.
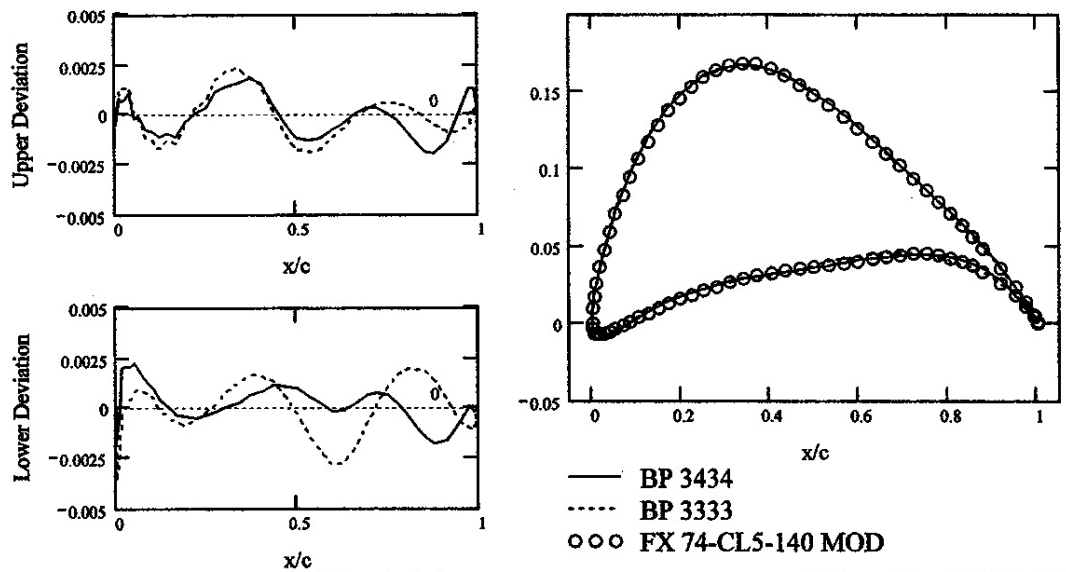

Figure 4: $\quad$ Low-speed FX 74-CL5-140 MOD airfoil. 


\subsection{Summary}

Each of the three parameterization methods was able to represent a high percentage of the sample airfoils. Of the 63 airfoils, the Bezier parameterization reproduced 58, BP 3333 reproduced 59, and BP 3434 reproduced 62. In most cases the approximation required less than 10,000 function evaluations. Typically, the Bezier approximations required the fewest function evaluations and the BP 3434 approximations required the most.

The biggest limitation of the Bezier approximations is the discontinuous second derivative at the camber and thickness crests which can result in inaccurate flow field simulations. A less significant difficult is the zero trailing edge thickness which is a problem for some airfoils such as the Eppler E 863.

The BP 3333 approximation has the least control point freedom which can be beneficial in some cases such as for the Eppler E 417. Two classes of airfoil cannot be successfully represented by BP 3333 ; those with a radical change in the trailing edge camber curve and those with a camber curve that dips below the $\mathrm{x}$-axis.

The BP 3434 approximation is the most robust due to the additional control points at the trailing edge. This can be a problem in that it can result in the camber crest being pushed to far back or premature convergence with the shapes with sharp edges of incorrect trailing edge directions.

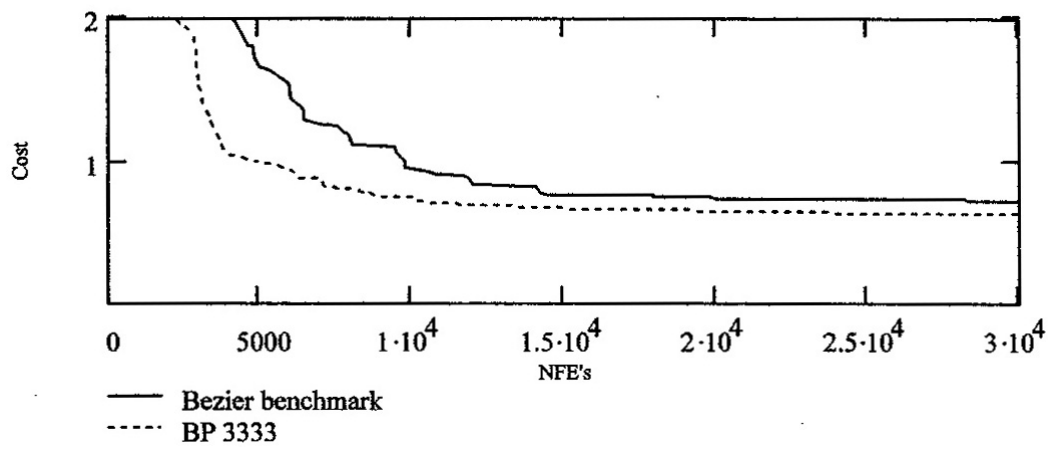

Figure 5: Effect on convergence.

\section{Effect of parameterization on design speed}

The previous discussion showed that all three parameterization methods can represent a broad range of airfoils. The next issue of concern is the affect of the parameterization on the robustness and rate of convergence for an inverse design problem. The focus is narrowed to a comparison between the Bezier and BP 3333 parameterizations. The results of an optimization to a $112^{\circ}$ cambered turbine blade will be presented here. The optimization was based on finding the target airfoil that best matches the known, theoretical, pressure distribution on an airfoil. This allows us to determine the robustness and convergence properties of the optimization process, while insuring that the optimum has been reached. 


\subsection{Convergence for a $112^{\circ}$ cambered turbine blade}

The convergence rate for the Bezier and BP 3333 parameterizations are shown below. Clearly the BP 3333 parameterization results in a more rapid convergence to the solution than the Bezier parameterization for this case, and was also found to be the case for a Liebeck design. However, similar convergence histories for Bezier and BP 3333 parameterizations occurred for a C4/70/C50 turbine blade target. Additionally, the BP 3333 approximation did a better job of converging to the target airfoil than the Bezier approximation.

\section{Conclusions}

The three parameterization methods are all capable of representing a wide range of airfoil shapes with a slight edge going to BP 3434. However, the BP 3333 parameterization performed better, and certainly no worse than the Bezier parameterization for inverse design.

\section{References}

[1] Abbot, I.H., and von Doenhoff, A.E., Theory of Wing Sections, Dover Publications, New York, 1959.

[2] Rogalsky, T., Derksen, R.W., and Kocabiyik, S., Differential Evolution in Aerodynamic Optimization, Canadian Aeronautics and Space Journal, 46(4), pp. 183-190, 2000.

[3] Sobieczky, H., Parametric Airfoils and Wings. Recent Developments of Aerodynamic Design Methodologies, ed. Fujii, K. and Dulikravich, G.S., Friedr. Vieweg \& Sohn Verlagsgesellschaft $\mathrm{mbH}$, Braunschweig/Wiesbaden, pp. 71-87, 1999.

[4] Sobiczky, H., Manual Aerodynamic Optimization of an Oblique Flying Wing, $36^{\text {th }}$ Aerospace Sciences Meeting \& Exhibit, Reno, NV, AIAA Paper 98-0598, 1998.

[5] Oyama, A., Obayashi, S., and Nakahashi, K. Fractional Factorial Design of genetic coding for aerodynamic optimization, AIAA Paper 99-3298, 1999.

[6] Rogalsky, T. Acceleration of Differential Evolution for Aerodynamic Design, Ph.D. Thesis, University of Manitoba, 2004.

[7] Abbot, I.H., and von Doenhoff, A.E., Theory of Wing Sections, Dover, New York, 1959.

[8] Eppler, R., Airfoil Design and Data, Springer-Verlag, Berlin, 1990

[9] Selig, M.S., Guglielmo, J.J., Broeren, A.P. and Giguère. P., Summary of Low-Speed Airfoil Data, Vol. 1, SoarTech Publications, Virginia Beach, 1995. 